\title{
Developing Methods to Predict Chemical Fate and Effect Endpoints for Use Within REACH
}

\author{
Kathrin Fenner ${ }^{* a, b}$, Silvio Canonicac ${ }^{c}$, Beate I. Escher ${ }^{d}$, Lukas Gasser ${ }^{a, b}$, Simon Spycher ${ }^{d}$, and \\ Holger C. Tülpa,b
}

\begin{abstract}
With the pending implementation of $\mathrm{REACH}$, both old and new chemicals will have to be registered and chemical safety reports will have to be compiled. Depending on the yearly tonnages produced or imported, (eco-) toxicological and chemical fate data of varying degrees of detail will have to be produced. It has been forecast that these new requirements will result in higher costs for registration and an increased need for animal testing. Some of this additional workload could be avoided by making use of in vitro or in silico prediction methods. At Eawag (Swiss Federal Institute of Aquatic Science and Technology) several research groups are working on the development and validation of quantitative structure-activity relationships (QSARs) and related methods to predict ecotoxicological and fate endpoints, such as reactivities in or partitioning between different environmental media, based on chemical structure or easily measurable physico-chemical properties. When developing such tools, special attention has to be paid to use only descriptors whose mechanistic significance for the modelled endpoint is well understood on a molecular level. In this article four examples of our work in the field of compound fate and effect predictions will be presented: i) the measurement of compound descriptors for use in linear-free-energy relationships to predict partition coefficients between environmental media; ii) the development of free-energy relationships for the prediction of indirect photolysis; iii) the evaluation of existing structure-biodegradability models to predict soil biodegradation half-lives; and iv) the application of mode-of-action-based test batteries to develop quantitative structure-activity relationships to classify chemicals according to their modes of toxic action.
\end{abstract}

Keywords: Biodegradability prediction - Chemical property prediction · Indirect phototransformation . Mode-of-action classification · Polyparameter linear-free-energy relationships $\cdot$ REACH

\section{Introduction}

The pending implementation of the new European chemicals regulation REACH (Registration, Evaluation and Authorization of Chemicals) [1] will have a significant impact on the development and validation of in silico methods, among them

\footnotetext{
*Correspondence: Dr. K. Fenner ${ }^{a, b}$

Tel.: +41448235085

Fax: +41448235471

E-Mail: kathrin.fenner@eawag.ch.

aEawag, Swiss Federal Institute of Aquatic Science and Technology

Department of Environmental Chemistry

Ueberlandstrasse 133

$\mathrm{CH}-8600$ Duebendorf

VInstitute of Biogeochemistry and Pollutant Dynamics ETH Zurich

$\mathrm{CH}-8092$ Zürich

'Eawag, Swiss Federal Institute of Aquatic Science and Technology

Department of Water Resources and Drinking Wate

dEawag, Swiss Federal Institute of Aquatic Science

and Technology

Department of Environmental Toxicology
}

(quantitative) structure-activity relationships ((Q)SARs [2]). One of the main cornerstones of REACH is that the distinction between old and new chemicals will disappear. In other words, all chemicals currently on the market as well as newly developed chemicals will need to undergo registration and, depending on the tonnage produced, a more or less extensive evaluation of their risk to humans and the environment ('chemical safety assessment'). As a consequence, the need for environmental fate data, i.e. data on partitioning and reactivities, as well as for (eco-)toxicological data will increase. Table 1 gives an overview of the data requirements for the various tonnage classes.

An estimated cost of 1 to 5 billion $€$ is expected due to required additional testing, primarily due to toxicity studies ( $h t t p: / / e u-$ ropa.eu.int/comm/enterprise/reach/whitepaper/ biajune2002.htm), with some industry groups projecting even higher costs (18-32 billion $€$ ) [3]. The implementation of REACH will thus significantly increase the need for developing and validating in vitro, in silico and read-across methods as cost-effective alternatives to determine chemical fate and effect endpoints. The Institute for Health and Consumer Protection (IHCP) of the European Commission issued several documents on the cost-saving potential of QSARs and estimated that 700-940 Mio $€$ and a lot of test animals could be saved [4]. However, they also acknowledged that there are still gaps for a full regulatory acceptance of most published QSARs, amongst them a vigorous and independent validation exercise.

While in the present Swiss [5] and European legislation the use of (Q)SARs and related predictive methods for fate and effect endpoints is still limited, REACH explicitly opens up the opportunity to predict such properties based on knowledge about structurally related compounds and based on the outcome of mechanism-based in vitro effect tests. Chapter 2, Annex VI of REACH states that "The registrant should also collect all other available and relevant information (...). This should include information from alternative sources (e.g. from 
Table 1. Relevant data requirements for the chemical safety report requested within REACH [1] for chemicals of different tonnages

Physico-chemical and environmental fate
data
$\geq 1$ t/a (Appendix VII) (standard data requirements)
Octanol-water partition coefficient

Water solubility

Vapour pressure

Ready biodegradability

$\geq 10$ t/a (Appendix VIII) (additional data requirements)

$\begin{array}{ll}\text { Hydrolysis as a function of } \mathrm{pH} & \text { Short-term toxicity testing on fish } \\ \text { Adsorption/desorption screening study } & \text { Activated sludge respiration inhibition test }\end{array}$

$\geq 100$ t/a (Appendix IX) (additional data requirements)

Dissociation constant in water

Long-term toxicity testing on daphnia

Simulation testing on ultimate degradation in surface water

Soil degradation simulation study

Sediment degradation simulation study

Identification of degradation products

Bioaccumulation in aquatic species (fish)

Long-term toxicity testing on fish

Reproductive toxicity to fish

Short-term toxicity to soil invertebrates

Effects on soil micro-organisms

Short-term toxicity to plants

Refined adsorption/desorption study

$\geq 1000$ t/a (Appendix X) (additional data requirements)

\section{Long-term toxicity testing on soil invertebrates}

Long-term toxicity testing on plants

Long-term toxicity to sediment organisms

Long-term or reproductive toxicity to birds

(Q)SARs, read-across from other substances, in vivo and in vitro testing, epidemiological data) which may assist in identifying the presence or absence of hazardous properties of the substance and which can in certain cases replace the results of animal tests." In Annex XI it is further specified that only those (Q)SAR models shall be used whose scientific validity has been established and which are adequately documented.

At Eawag (Swiss Federal Institute of Aquatic Science and Technology) one of the three major fields of research activities, besides 'urban water management' and 'aquatic ecosystems', is 'chemicals and their effects in water'. Within the latter field, ongoing research is focused in five working groups (Table 2), whose activities reach from fundamental research on ecotoxicological mechanisms and chemical fate processes (WG 1-3) all the way to putting group is to develop and validate methods to predict fate and ecotoxicological endpoints (Swiss Federal Institute of Aquatic Science and Technology)

\section{Working group 1 \\ Realistic exposure scenarios}

Working group 2

Internal exposure

Working group 3 of large sets of diverse chemicals based on chemical structure, easily measurable physico-chemical properties or in vitro assays.

When developing such methods, a number of processes need to be understood on a molecular level (e.g. adsorption, bulk partitioning, or complexation; direct or indirect (photo)chemical, or biologically mediated transformation reactions; uptake into organisms and interaction with biological target molecules). The quantification of these individual processes requires quantitative information on the pertinent compound- and system specific properties and reactivities. For some of the abiotic processes such as partitioning between bulk phases, adsorption to surfaces, or degradation reactions such as hydrolysis or direct photolysis, the underlying molecular mechanisms are fairly well understood and quantifiable, and various predictive methods for these endpoints already exist. Unfortunately, many of these methods are based on regressions against various types of molecular descriptors that are often selected on purely statistical grounds, rather than on a mechanistic understanding of their influence on the endpoint of interest. As a consequence, it is often not clear to what range of chemical structures they are applicable and they are mostly valid for one specific endpoint only. One of the common denominators of the tools developed in our research is that they are based on descriptors or explanatory variables whose mechanistic significance for the modelled endpoint is well understood. In such a way, we assure that the tools are broadly applicable to diverse sets of chemical structures, that their applicability domain is clearly defined, and that they can encompass several related endpoints in a consistent manner. Specific activities in this area include the measurement of compound descriptors for use in polyparameter linear-free-energy relationships to predict partition coefficients between various environmental media (Section 2), and the development of free-energy relationships for the prediction of indirect photolysis (Section 3).

Whereas for the quantification of abiotic processes it is sufficient to understand the chemicals' tendency to interact with a given sorbent or reactant and to know the

Table 2. Working groups (WG) within field of activity 'chemicals and their effects in water' at Eawag

Multiple effects, multiple stressors and sequential exposure

Working group $4 \quad$ Environmental risk assessment of chemicals

Working group $5 \quad$ Decision support for government agencies, politics, and industry 
abundance and properties of that sorbent or reactant, the situation becomes considerably more complex when biological processes are involved. To quantify biodegradability, for instance, the bioavailability of the compounds, their interaction with enzyme systems catalyzing their breakdown and the abundance of these enzyme systems further need to be understood. Our work on validating and analyzing the shortcomings of existing structure-biodegradability models to predict biodegradation rates in soil (Section 4) confirms that the most we can currently expect from these models is that they correctly rank chemicals with regard to their relative tendency to be biodegraded, whereas prediction of actual biodegradation rates in real environments is currently not feasible. Likewise, to predict ecotoxicological effects in a mechanistically sound manner, understanding bioavailability and identifying the targets and key mechanisms of toxic effects are essential prerequisites. The application of mode-of-action-based test batteries to develop quantitative structure-activity relationships to classify chemicals according to their modes of toxic action (Section 5) is an example of our research in this area.

The four areas of research mentioned will be presented in more detail in the following and it will be pointed out how and at what point of the assessment process the resulting models might be instrumental for chemical safety assessment within REACH.

\section{A General Principle to Predict Partitioning Between Diverse Environmental Phases}

According to REACH, a detailed exposure assessment for the five environmental spheres, aquatic, terrestrial and atmospheric environment, food chain and sewage treatment plants, needs to be carried out for chemicals that are produced in volumes above $10 \mathrm{t} / \mathrm{a}$ and that are dangerous in accordance with $67 / 548 / \mathrm{EEC}$ or $1999 / 45 / \mathrm{EC}$ or that have been demonstrated to exhibit PBT or vPvB properties. Chemicals are subject to various partitioning processes within and between these environmental spheres, which influence their expected presence in any of these spheres. The measurement of these partition coefficients is tedious and coefficients reported in the literature often vary over several orders of magnitude for a given compound and partitioning system [7].

Alternatively, many different predictive models for partition coefficients exist, ranging from one parameter linear-free-energy relationships, over structure-property relationships all the way to quantum chemical calculations. Most of them, however, suffer from one of the following shortcomings: i) they only apply to compounds with similar molecular structures, ii) they fail in describ-

ing complex environmental phases such as natural organic matter, where possible interaction sites are countless and not well characterized, or iii) they are developed for one specific partitioning system only and do not produce coefficients that are consistent over several systems, e.g. in terms of the thermodynamic cycle. Polyparameter linearfree-energy relationships (pp-LFER), which describe the partitioning of a chemical between two phases in terms of the energy contributions of the most important solute-phase intermolecular interactions, overcome these problems and are therefore rapidly gaining ground in environmental chemistry [8] [9] One of the most prominent pp-LFERs for describing equilibrium partitioning between bulk phases is the solvation parameter model by Abraham [10] (Eqn. (1)).

$\log K=e E+s S+a A+b B+v V$

The dependent variable $\log K$ is the logarithm of the partition coefficient and the five descriptor pairs quantify the molecular interactions that govern the partitioning process: Van der Waals interactions $(e, E)$, polar interactions $(s, S), \mathrm{H}$-bond donor $(b, A)$ and acceptor $(a, B)$ interactions and cavity formation in bulk media $(v, V)$. The five descriptors in capital letters describe the tendency of a given chemical to undergo these types of interactions independent of the partitioning system in question (solute descriptors). The corresponding descriptors in lower case letters describe the difference in capacity between any two phases to undergo the various intermolecular interactions (phase descriptors). Similar equations for adsorption to surfaces exist, the main difference being that there is no need for the cavity formation term [11]. Due to the fundamentally mechanistic nature of the solute descriptors, they are universally applicable to all relevant partitioning systems and therefore only need to be measured once. The same is true for the phase descriptors. Once measured, they are valid to quantitatively describe the partitioning behaviour of all sorts of neutral compounds. Thus, if both solute and phase descriptors are known, the equilibrium partitioning of a chemical in a two-phase system can be calculated with much higher accuracy than with other methods. A further advantage of the mechanistic basis of pp-LFERs is that the interpretation of phase descriptors and solute descriptors is straightforward and allows for efficient consistency checking of experimentally obtained values.

Phase descriptors for numerous bulk phase-water, bulk phase-air and surface-air partitioning systems have been and are currently being determined experimentally [1220]. A selection of environmentally relevant partitioning systems with available phase descriptors is given in Table 3 . It should be noted that especially partitioning between air and bulk phases or surfaces is highly dependent on temperature and, in the latter case, also humidity. Phase descriptors have therefore always been determined under various conditions of temperature and humidity and in some cases expressions for these dependencies on environmental conditions could even be derived on theoretical grounds [11]. The availability of phase descriptors for a broad variety of environmental phases such as snow, mineral surfaces, soot etc. and conditions opens up an avenue to describe the effect of the variable composition of relevant environmental subcompartments such as soil solid material or tropospheric aerosols on partitioning much more concisely than it is currently done in chemical fate modelling [21].

To pave the way towards practical applicability of pp-LFERs, there is not only a need to determine phase parameters for the relevant environmental systems but there is also a need for methods to efficiently determine solute descriptors for a broad set of compounds. Especially for polar compounds with multiple functional groups such as pesticides and veterinary pharmaceuticals there is currently a lack of descriptors. While the $E$ and the $V$ descriptors can be calculated precisely from molecular structure, the $S, A$ and $B$ descriptors have to be determined experimentally. In our research group, solute descriptors for complex environmental chemicals are currently being measured using high-performance liquid chromatography systems (HPLC). This method is based on the fact that the measured net retention time of a given compound in an HPLC system is proportional to its partition coefficient

Table 3. Environmentally relevant two-phase systems with available phase descriptors

Two-phase system

Reference

Surface-air partitioning

Quartz-air

Water-air

Snow-air

Diesel soot-air

Humic acid-air

Bulk phase partitioning

Water-soil organic matter

Water-blood

Water-fatty tissues

\section{Passive transport}

Cell permeation

Plant cuticle permeation 
in the system. To determine the three missing solute descriptors $S, A$ and $B$, at least three HPLC systems are therefore required. These systems must differ strongly in the intermolecular interactions that control the retention (partitioning) process. For the exact determination of solute descriptors we actually use nine different HPLC systems that include various mobile and stationary phases. With this setup we recently measured solute descriptors for a set of about 40 substances from different classes of pesticides and pharmaceuticals. When inserted into existing pp-LFER equations for partition coefficients such as $\log K_{\text {ow }}$ or $\log K_{\text {oc }}$ and compared to directly measured values of these partition coefficients, we could show that partition coefficients derived with solute descriptors determined in the HPLC systems did not exhibit any systematic error and deviated from directly measured ones by a factor of 3 on average only. It can thus be expected that the same solute descriptors can be used to predict other partitioning properties with a similar level of accuracy.

In the context of REACH, pp-LFERs offer the opportunity to predict various types of partitioning properties and transfer rates based on only five solute descriptors and in a consistent manner. At the moment, their applicability is limited by the lack of solute descriptors and also phase descriptors for some environmentally relevant systems such as soils or sediments. While experimental determination of phase and solute descriptors is ongoing in different research groups, another option to obtain solute descriptors, besides the straightforward HPLC method mentioned, is to predict them with a group contribution approach [22]. This approach has been implemented as the so-called 'Absolv' module in the ADME-Boxes software (C) 2001-2006, Pharma Algorithms, Inc.). Although the predictive power of 'Absolv' is increasing, the need for experimentally determined solute descriptors is currently still strong as a means to validate and improve 'Absolv' by expanding the testing or training database. Currently, the use of 'Absolv'-generated solute descriptors cannot yet be recommended due to a lack of transparency with regard to the calculation algorithm and its validity [23]. In the future, however, it could become the method of choice to predict partition coefficients.

\section{Prediction of Indirect Phototransformation in Surface Waters}

As defined by the requirements listed in Table 1, the tendency of chemicals to be removed from the environment by abiotic or biotic degradation has to be documented for their registration. For chemicals with a production volume $<100 \mathrm{t} / \mathrm{a}$, hydrolysis is explicitly stated as the only abiotic chemical process to be studied. However, for certain compounds other processes, such as reductions, oxidations or photoinduced reactions, may constitute other relevant abiotic pathways leading to degradation. Here, we give an overview of the importance of different photoinduced reactions and suggest a simple approach to assess the potential for indirect phototransformation through aquatic photooxidants. This section might help registrants to evaluate the photodegradation potential of a chemical to be registered, and could serve as a basis for proposing photodegradation studies in the testing proposal for a given chemical.

Rates of direct phototransformation are generally highly dependent on the electronic absorption spectrum of the target contaminant and the quantum yield of its reaction, the latter quantity being difficult to predict by computational methods. A well studied example, where direct phototransformation in surface waters is a relevant environmental removal process, are fluorescent whitening agents, a class of high production volume chemicals widely used in laundry detergents and paper and cloth manufacturing [24-27]. A limited number of QSARs concerning very specific photoreactions, such as the photohydrolysis of aromatic halides [28], have been derived to date.

A different type of phototransformation reactions are indirect (or sensitized) phototransformations, which occur mainly by interaction between target contaminant and transient reactants formed upon irradiation of light-absorbing water components, such as dissolved natural organic matter (DOM), nitrate and nitrite ions, or iron(III) complexes. Relevant transient reactants occurring in the aerated, sunlightexposed upper layer of surface waters are called photooxidants and comprise hydroxyl radicals, carbonate radicals, singlet molecular oxygen, excited triplet states of DOM ( ${ }^{3}$ DOM $\left.{ }^{*}\right)$ and other DOM-derived radicals [29]. The overall first-order rate constant for depletion by indirect phototransformation is made up of the contribution of each individual photooxidant. Hydroxyl radicals are among the most powerful oxidants occurring in the aquatic environment and react with most organic compounds containing aromatic moieties very readily, at nearly diffusion-limited rates. QSARs for aqueous hydroxyl radical reaction rate constants have been recently reviewed [30]. Such rate constants are available for more than one thousand compounds [31] and can be easily determined using competition kinetics methods [32]. However, the very low concentration of $\mathrm{OH}$ radicals in sunlit natural waters, which typically results in half-lives in the order of several months for this specific type of reaction, reduces their importance for the degradation of aquatic contaminants.

The other known aquatic photooxidants are more selective than hydroxyl radicals, which means that their second-order rate constants vary by several orders of magnitude and high reactivity is reached for a limited number of compounds only. However, for such subgroups of compounds they can represent significant causes of degradation, since their concentration may be much higher than that of hydroxyl radicals. ${ }^{3} \mathrm{DOM}^{*}$ seems to play a dominant role in the surface water degradation of electron-rich phenolic compounds [33] and various phenylurea herbicides [34] Therefore interest has grown in quantifying this indirect photochemical pathway and predicting degradation rates of target contaminants. Because the nature of ${ }^{3} \mathrm{DOM}^{*}$ is largely unknown and, due to the great variety of chromophoric DOM components, their characterization by spectroscopic techniques is currently not feasible, we have used model aromatic ketones to mimic DOM triplet states. For substituted phenols [35] and, more recently, phenylureas [36] we could show that oxidation of such target contaminants was largely controlled by the rate of bimolecular electron transfer to the excited triplet state of the aromatic ketone. Second-order rate constants for the initial electron transfer from a target phenol to excited triplet ketones followed a non-linear relationship (Fig. 1), which could be modelled using a Marcus or a Rehm-Weller relationship [33], with the Gibbs free energy of electron transfer $\left(\Delta G_{\mathrm{el}}^{\mathrm{o}}\right.$ ) between the oxidizing species and the contaminant being the descriptor variable. This relationship allows the prediction of absolute rate constants if the one-electron standard reduction potentials of the oxidizing species (the excited triplet) and of the target contaminant are known. Fig. 1 shows that rate constants ( $k^{\mathrm{obs}}$ ) level off towards negative $\Delta G_{\mathrm{e}}^{\circ}$, approaching limiting values of $\approx 4 \times 10^{9} \mathrm{M}^{-1} \mathrm{~s}^{-1}$, which can be considered as the diffusion limit for this specific reaction, and that they strongly decrease with increasing $\Delta G_{\mathrm{e} \text {. }}^{\mathrm{o}}$.

For fast-reacting compounds such as electron-rich phenols, half-lives of the order of a few hours are typical for surface waters [33]. These are about two orders of magnitude shorter than those determined for the hydroxyl radical-induced degradation [37], which may be considered a slow, unspecific 'background' degradation for those compounds showing reactivity towards ${ }^{3} \mathrm{DOM}^{*}$. The relationships derived to date for substituted phenols and phenylureas allow us to select, based on their one-electron standard reduction potential, candidate contaminants that will probably 


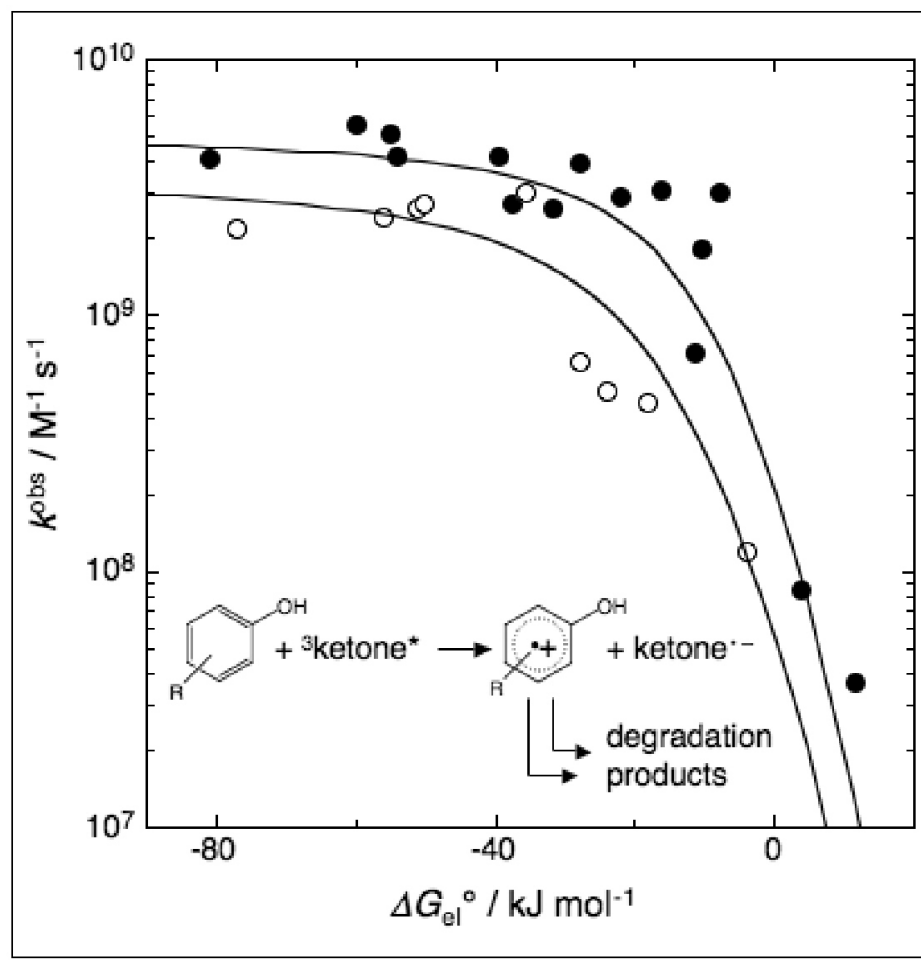

Fig. 1. Rehm-Weller plots for electron transfer rate constants from substituted phenols $\left(k^{\text {obs }}\right)$ to 1) excited triplet benzophenone and 2-acetonaphthone (full circles) and 2) excited triplet 3'methoxyacetophenone (open circles). Lines correspond to fits of the two different data sets to the Rehm-Weller equation [33].

undergo indirect photolysis by the ${ }^{3} \mathrm{DOM}^{*}$ mechanism. Based on the estimated effective potential of DOM, we can further predict that for compounds having one-electron standard reduction potentials above a certain limit, ${ }^{3} \mathrm{DOM}^{*}$-induced phototransformation should be negligible. We believe that once the method has been verified for other classes of compounds and refined accordingly, it will be possible for a given target contaminant either to quantify its ${ }^{3} \mathrm{DOM}^{*}$-induced phototransformation or to exclude it a priori. In the context of REACH such a method will be helpful to estimate the likelihood that, for a given contaminant, indirect phototransformation besides hydrolysis plays a role for its abiotic degradation in surface waters.

\section{Evaluation of QSARs for Biodegradability}

For compounds that do not contain an easily hydrolysable moiety or that either have a low potential for phototransformation or are not exposed to sunlight, biodegradation is often the only remaining pathway removing them from the environment. For PBT (Persistence, Bioaccumulation and Toxicity) assessment as well as for more detailed exposure analyses, the half-lives of a compound in soil and water are essential compound properties that need to be known. Within REACH, cutoff criteria are given for persistent compounds (soil half-life $>120 \mathrm{~d}$, aqueous half-life $>40 \mathrm{~d}$ (fresh water) and $>60$ $\mathrm{d}$ (marine water), marine sediment half-life $>180 \mathrm{~d}$ ) and for very persistent compounds (soil and sediment half-life $>180 \mathrm{~d}$, aqueous half-life $>60 \mathrm{~d}$ ). Several initiatives have been taken to identify molecular substructures and descriptors that influence biodegradability and to construct quantitative models for biodegradability prediction based thereupon. To date, a set of validated models that allow for a reasonably accurate prediction of ready biodegradability is available [38]. However, for comparison to cutoff values and for exposure modelling, a more precise prediction of environmental half-lives in the media soil, water and sediment is necessary. Developing QSAR models that fulfill this purpose seems to resemble the quest for the Holy Grail, as a series of continuously less optimistic editorials about the prospect of predicting biodegradation rates over a period of 20 years illustrates [39-41].

Currently, the most frequently used models for the prediction of primary or ultimate biodegradation half-lives are the BIOWIN Primary or Ultimate Survey Models from the US EPA EPI Suite package (freely downloadable from http://www.epa.gov/opptintr/ exposure/docs/episuite.htm). They are based on the results of an expert survey and use a group contribution approach to predict biodegradability on a scale from 1 to 5 . To convert this raw output into compartmental half-lives, it is suggested in the EPI Suite package that the results from the BIOWIN Survey Models be translated into water halflife categories $(<1.75$ : $180 \mathrm{~d}, 1.75-2.25: 60$ d, 2.25-2.75: $37.5 \mathrm{~d}, 2.75-3.25: 15 \mathrm{~d}, 3.25-$ 3.75: $8.7 \mathrm{~d}, 3.75-4.25: 2.3 \mathrm{~d}, 4.25-4.75: 1.3$ d, $>4.75: 0.2 \mathrm{~d}$ ) and that soil and sediment half-lives be derived from these water halflives by using multiplication factors of 2 and 9 respectively. Alternatively, Arnot et al. [42] suggest a regression equation for translating BIOWIN raw output into half-lives, which they have derived from experimental soil and water half-lives of a set of 40 diverse chemicals.

In the context of our work on the exposure assessment of pesticide transformation products [43], we evaluated how well soil half-lives could be predicted with the BIOWIN Primary Survey Model (PSM). To translate the raw PSM output into half-lives we used the translation rules suggested in the EPI Suite package, the regression equation by Arnot et al. [42] as well as our own regression equation fitted to our collection of pesticide data (comprising 38 compounds with experimental soil half-lives, including 20 parent pesticides and 18 pesticide transformation products). Where more than one soil half-life was reported for a given compound, the median was used in the comparison. Fig. 2 shows how the BIOWIN PSM output compares to the experimental soil half-lives, and how the EPI Suite translation rules and the two regression equations compare to the data points.

From the comparison in Fig. 2, it can be seen very clearly that the output of BIOWIN PSM and the median experimental half-lives do not correlate well $\left(r^{2}=0.49\right)$. Our regression equation is mainly driven by a few old, recalcitrant pesticides such as DDT, heptachlor, aldrin and dieldrin in our data set. For the better degradable pesticides with BIOWIN outputs between 2.5 and 4 , no correlation between experimental soil half-lives and BIOWIN output can be detected at all. In Table 4 these results are summarized as the maximum negative and positive deviations, the average deviation as well as the rms error between experimental and predicted soil half-lives on a log scale. It clearly shows that none of the three translation methods, i.e. the EPI Suite translation rules, the Arnot et al. correlation, or our own correlation, is superior. All three methods yield maximal errors of around $1.5 \log$ units, which translate into maximal uncertainties in the prediction of half-lives of a factor of 30 . While EPI Suite translation rules show a tendency to underpredict half-lives, using the Arnot et al. relationship rather seems to lead to an overprediction of half-lives for the 30 compounds investigated.

These large uncertainties are quite unsatisfactory and will translate into correspondingly large errors in the exposure assessment of compounds for which no experimental half-lives are available. Since REACH explicitly allows for read-across, we tested whether, for the special case of transformation products, the predictions could be optimized by using the parent pesticide half-life as a starting point [44]. Unfortunately this approach also failed to improve the predictive power significantly.

All in all, using BIOWIN for the prediction of compartmental half-lives results in maximal uncertainties as large as a fac- 


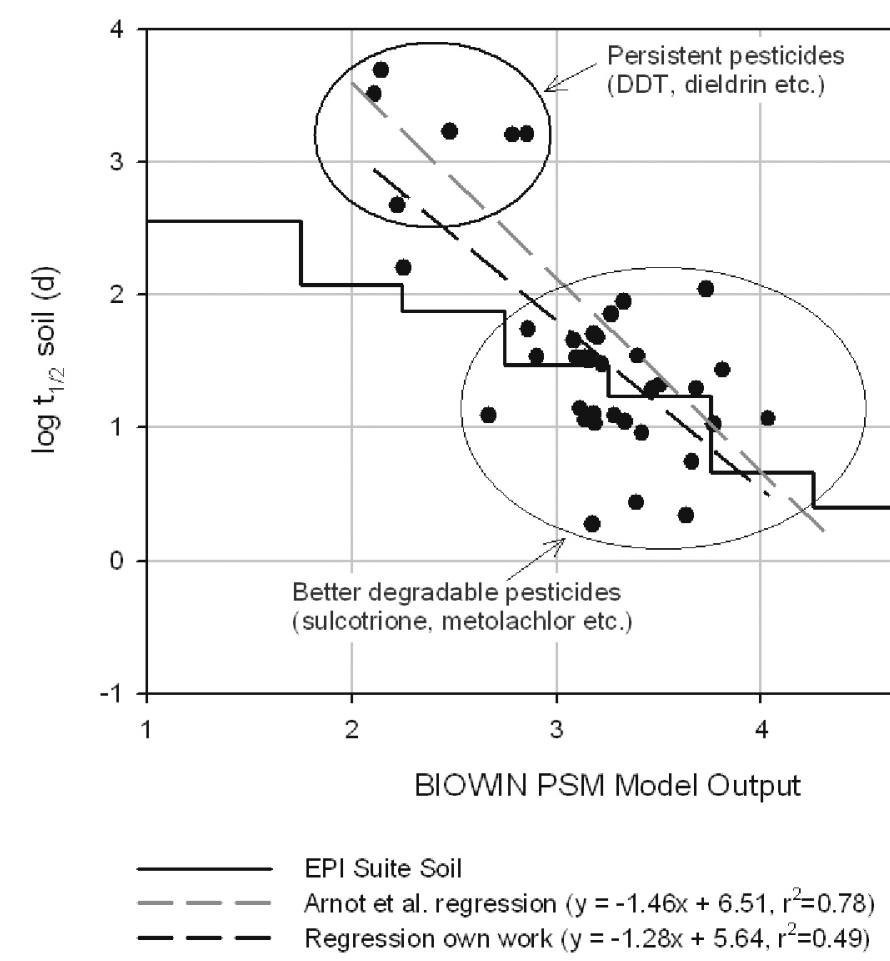

Fig. 2. Comparison of BIOWIN PSM output with experimental soil half-lives for 30 pesticides and their transformation products. In addition, three possible methods for translating BIOWIN PSM output into actual half-lives are also indicated: EPI Suite translation rules (indicated as 'EPI Suite Soil'), the Arnot et al. [42] regression and our own regression based on the pesticide data.

tor of 30 and mean uncertainties, deduced from the rms error, of about a factor of 5 , which is hardly sufficient for an in-depth exposure analysis. Fig. 2 indicates that the predictive power might be sufficient to distinguish between clearly persistent and clearly non-persistent compounds for the purpose of PBT assessment, which is confirmed by a similar analysis by Aronson et al. [45]. Actual biodegradation rates in soil or water, however, are influenced by many different factors beside chemical reactivity such as temperature, humidity, $\mathrm{pH}$, soil texture, pollutant concentration, redox conditions and most importantly the microbial consortium present. They can therefore not be expected to be predictable based on results from ready biodegradability tests or from expert surveys, which are the basis of
BIOWIN. In the future, models that allow for a more accurate prediction of half-lives will only be obtained through training on actually measured half-lives. In doing so, the challenge lies in separating the influence of molecular structure on biochemical reactivity from the confounding influences of different environmental and experimental conditions under which biodegradation rates have been measured [46].

\section{Development of QSARs for Ecotoxicological Endpoints}

In the field of QSARs for toxic endpoints we face a paradoxical situation today with both the need for QSARs as well as scepticism against their usefulness increas-

Table 4. Maximum negative and positive deviations, the average deviation as well as the rms (root mean square) error between experimental and predicted soil half-lives for the three translation methods shown in Fig. 2, as well as for two read-across methods (parent compound (PC) adjusted regressions). All differences are expressed on a log scale $\left(\log t_{1 / 2, \text { soil }}(d)\right)$

$\begin{array}{lllll}\log t_{1 / 2, \text { soil }}(d) & \text { Average deviation } & \begin{array}{l}\text { Maximum } \\ \text { positive } \\ \text { deviation }\end{array} & \begin{array}{l}\text { Maximum } \\ \text { negative } \\ \text { deviation }\end{array} & \text { rms error } \\ \text { BIOWIN } & -0.20 & 1.21 & -0.73 & 0.73 \\ \text { Regression Arnot } & 0.29 & 1.60 & -0.98 & 0.67 \\ \text { Regression own work } & 0.00 & 1.31 & -1.22 & 0.59 \\ \text { PC adjusted regression Arnot } & -0.27 & 1.44 & -1.35 & 0.62 \\ \text { PC adjusted regression own work } & -0.26 & 1.44 & -1.32 & 0.61\end{array}$

ing. The reasons and possible solutions to this problem are discussed in a very clearsighted article by Veith [47]. He states that to overcome this scepticism, we first need to understand mechanisms in order to apply a model correctly and, second, we need to move from the current 'test now-model later' approach to using QSARs for setting the priorities for experimental testing. Both claims fit in with the research conducted at Eawag over the last decade, as we will try to show here. Currently, the technical guidance document [6] contains a chapter on QSARs for ecotoxicity endpoints such as acute toxicity towards fish and other aquatic life. Unfortunately, at present, in the EU QSARs are accepted only for the prediction of baseline toxicity. Pollutants that are hydrophobic and persistent are a particular problem because they tend to bioaccumulate. Inside the organisms they accumulate preferentially in storage lipids and in membrane lipids. Membrane lipids are target sites for toxic effects, with baseline toxicity being a nonspecific disturbance of structure and functioning of the lipid bilayer of membranes [48]. Baseline toxicity, also called narcosis, constitutes the minimum toxicity of any compound. Therefore it is useful to be able to estimate the effect level for baseline toxicity. However, in order to identify 'pollutants of concern', it is desirable to have tools for the identification and prediction of chemicals with specific, and therefore more potent, modes of toxic action (MOA). Since identification of MOA and classification are crucial, we discuss these issues in more depth before presenting an example of a predictive model from our own research.

\subsection{Classification}

The most relevant MOA-based QSAR collection is the ASTER-system (ASsessment Tool for Evaluating Risk) of the US EPA. In ASTER, the appropriate QSAR for a new compound is selected on the basis of the occurrence of chemical fragments [49]. However, a fragment-based rule system is limited because it reduces a chemical structure to a specified substructure and ignores the other topological and electronic features of the entire compound, which may influence its propensity to act according to a given mode of toxic action [50]. With the aim to overcome this limitation several studies on MOA classification have been published in the last years [51-53].

The comparison of these models reveals great differences in the number and types of MOAs considered, methods used to assess the predictive power, and the selection of the compounds. Already the experimental data used to build the models show fundamental differences in the assignment of MOAs with disagreements ranging from $23-32 \%$ within sets of overlapping compounds 
[54]. Furthermore, a general trend can be observed that the number of correctly classified compounds decreases rapidly with increasing diversity of the data sets. Thus, the coverage of different chemical classes is still a major problem in QSAR modelling and is only feasible if the variables of a model are closely related to the underlying molecular mechanism of toxicity [55].

An example from our work may serve to illustrate the complexity of MOA assignments. We have developed a set of bioassays to classify and to describe the toxicity of electrophilic chemicals [56]. The effects were related to reaction rate constants towards model nucleophiles [54]. However, the entire data set needed to be broken down into a number of different subsets because of distinct differences in the reaction mechanism of the nucleophilic substitution reaction and differences in preferred target nucleophile, which resulted in very small data sets and a large number of different QSAR equations unsuitable for further use in regulatory applications [57].

This example illustrates that there is still a long way to go until classification methods based on molecular descriptors can be applied for regulatory purposes, which require robust and particularly transparent models. Therefore it seems that in the first phase of $\mathrm{REACH}$ more pragmatic approaches based on the occurrence of fragments, like in ASTER or as proposed by von der Ohe et al. [58], will be used with the known limitations described above.

\subsection{Quantitative Models}

While the EU technical guidance document [6] only suggests QSARs for baseline toxicity, the OECD proposes one additional QSAR for the MOA of uncoupling of oxidative phosphorylation [59]. However, the assignment of chemicals to the appropriate QSAR is not complete nor fully correct according to our own analysis [60]. For assessing uncoupling, we have developed an in vitro test system based on time-resolved spectroscopy of single-turnover events in the photosystem of the photosynthetic bacterium Rhodobacter sphaeroides, which allows one to quantify baseline toxicity, uncoupling and inhibition of the electron transfer chain and the ATP synthetase and to differentiate between these mechanisms [61][62]. The results for uncoupling obtained with this test system have been shown to correlate quite well with other in vitro endpoints and cytotoxicity tests [61]. We also found linear correlations of the in vitro data with fish toxicity data, but those are of lower quality due to additional toxicokinetic parameters determining the overall effect in fish [61]. Nevertheless, the results obtained with this test system are a good example for the assignment of mechanisms of membrane toxicity and intrinsic toxicity to a large set of environmental pollutants.

The data on baseline toxicity and uncoupling (for a compilation see [60]) were used to develop a MOA classification scheme and to derive a QSAR for intrinsic uncoupling activity [63]. The classification scheme distinguishing uncouplers from other MOA [54] is based on specifying ranges of physico-chemical descriptors that are characteristic for uncouplers and, thus, it does not suffer from the drawbacks of, for example, fragment-based approaches. The striking feature of the quantitative model is its mechanistic basis and the low number of only three calculated descriptor variables. The mechanistic insights leading to such models might require years of experimental work, however, if such knowledge is available, it is invaluable to QSAR-modelers in order to focus their search for meaningful molecular descriptors and also to the regulatory agencies, who need transparent models as the basis for their decisions.

However, the work presented here is only a first step towards establishing QSARs for regulatory purposes, which need to fulfill stringent validation criteria. The OECD principles of (Q)SAR validation read as follows [64]: "To facilitate the consideration of a (Q)SAR model for regulatory purposes, it should be associated with the following information: (1) a defined endpoint, (2) an unambiguous algorithm, (3) a defined domain of applicability, (4) appropriate measures of goodness-of-fit, robustness and predicitivity, (5) a mechanistic interpretation, if possible". To fulfill point (4) and to expand and define point (3), a 'critical mass' of measured data is required, which goes beyond the work for developing an in vitro test. Financing for additional research to fill this data gap is difficult to obtain from national and international research foundations due to its lack of being fundamentally new. Nevertheless, we feel the obligation as a governmental research institution to make a contribution, not only for scientific advancement but also for application of our results in the context of $\mathrm{REACH}$ and the Swiss legislation.

\section{Conclusions}

The OECD principles of (Q)SAR validation request "a mechanistic interpretation [of the (Q)SAR], if possible". In our opinion, it goes without saying that QSARs intended for use in REACH must be defendable based on solid scientific findings, which is achievable exclusively through a mechanism-based algorithm. A mechanism-based QSAR brings with it further advantages that should also be of interest in the context of REACH: It is broadly applicable to structurally diverse sets of chemicals as well as for the prediction of several endpoints in a con- sistent manner, which has been illustrated by the example of $p p$-LFERs for the prediction of partition coefficients. While QSARs that fulfill this request exist for physicochemical properties and abiotic reactivities, biological processes such as ecotoxicological effects and biodegradation are generally considered too complex to be approached in such a way. It is encouraging that modeof-action-based classification has by now been recognized as an important basis for the development of stringent QSARs in that it helps decomposing observed effects into their several underlying processes, which, in turn, is the basis for successful modelling. In biodegradation research, however, two extremes are still observed: QSARs are either applicable to broad sets of different chemicals but can hardly be interpreted in a mechanistic manner, or their mechanistic basis is understood but they are only applicable to a very restricted set of chemicals structures. Future work in the field needs to go toward bridging this gap. Assigning chemicals to their most likely 'mode of enzyme-catalyzed reaction' and to develop QSARs for comparative assessment within these reaction classes might be a way forward.

\section{Acknowledgments}

Funding of the prevalidation study for the MOA classification scheme and the QSAR for intrinsic uncoupling activity by the Swiss-based 3R Research Foundation, as well as funding of the QSBR validation studies by the Swiss Federal Office for the Environment are gratefully acknowledged. The authors further thank René P. Schwarzenbach for helpful comments on the manuscript and Kai-Uwe Goss for sharing his knowledge on pp-LFERs.

Received: August 14, 2006

[1] EU, Council of the European Union, 2006: Common position adopted by the Council with a view to the adoption of a Regulation of the European Parliament and of the Council concerning the Registration, Evaluation, Authorisation and Restriction of Chemicals (REACH), establishing a European Chemicals Agency, amending Directive 1999/45/EC of the European Parliament and of the Council and repealing Council Regulation (EEC) No 793/93 and Commission Regulation (EC) No 1488/94 as well as Council Directive 76/769/EEC and Commission Directives 91/155/EEC, 93/67/EEC, 93/105/EC and 2000/21/EC. Brussels, available at: http://register.consilium.europa.eu/pdf/en/06/st07/st07524. en06.pdf (accessed 24-07-2006).

[2] In this article, the term '(Quantitative) Structure-Activity Relationship' ((Q)SAR) is used for all types of relations between a chemical structure and any of its fate (e.g. reactivity or partitioning properties) or ecotoxicological (e.g. EC50 values) endpoints. 
[3] J.G. Hengstler, H. Foth, R. Kahl, P.J. Kramer, W. Lilienblum, T. Schulz, H. Schweinfurth, Toxicology 2006, 220, 232.

[4] F. Pedersen, J.H.M. De Bruijn, S. Munn, C.J. Van Leeuwen, 'Assessment of additional testing needs under REACH. Effects of (Q)SARs, risk based testing and voluntary industry initiatives', European Commission, Editor, 2003, IHCP Institute of Health and Consumer Protection IHCP: Ispra, Italy.

[5] Switzerland has implemented a new legislation for chemicals in August 2005, which is harmonized with the EU directives of 1993 and the associated technical guidance document of 2003 [6], but it is expected that Switzerland will continue harmonization after the implementation of REACH.

[6] European Commission,TechnicalGuidance Document in Support of Commission Directive 93/67/EEC on Risk Assessment for New Notified Substances, Commission Regulation (EC) No 1488/94 on Risk Assessment for Existing Substances, and Directive 98/8/EC of the European Parliament and of the Council Concerning the Placing of Biocidal Products on the Market, 2003, Office for Official Publications of the European Communities, Luxembourg.

[7] R. Renner, Environ. Sci. Technol. 2002, 36, 410A.

[8] K. Breivik, F. Wania, Environ. Sci. Technol. 2003, 37, 4934.

[9] K.U. Goss, R.P. Schwarzenbach, Environ. Sci. Technol. 2001, 35, 1.

[10] M.H. Abraham, Chem. Soc. Rev. 1993, 22, 73.

[11] K.U. Goss, Critical Reviews in Environmental Science and Technology 2004, 34, 339.

[12] M.H. Abraham, P.K. Weathersby, J. Pharm. Sci. 1994, 83, 1450.

[13] K.U. Goss, R.P. Schwarzenbach, J. Colloid Interface Sci. 2002, 252, 31.

[14] C. Niederer, K.U. Goss, R.P. Schwarzenbach, Environ. Sci. Technol. 2006, in press.

[15] J.A. Platts, M.H. Abraham, Environ. Sci. Technol. 2000, 34, 318 .

[16] J.A. Platts, M.H. Abraham, A. Hersey, D. Butina, Pharm. Res. 2000, 17, 1013.

[17] S.K. Poole, C.F. Poole, J. Chromat. A 1999, $845,381$.

[18] C.M. Roth, K.U. Goss, R.P. Schwarzenbach, J. Colloid Interface Sci. 2002, 252, 21.

[19] C.M. Roth, K.U. Goss, R.P. Schwarzenbach, Environ. Sci. Technol. 2004, 38, 4078.

[20] C.M. Roth, K.U. Goss, R.P. Schwarzenbach, Environ. Sci. Technol. 2005, 39, 6632.

[21] C.W. Goetz, M. Scheringer, M. MacLeod, C.M. Roth, K. Hungerbühler, Environ. Sci. Technol. 2006, submitted.

[22] J.A. Platts, D. Butina, M.H. Abraham, A. Hersey, J. Chem. Inform. Comput. Sci. 1999, 39, 835 .

[23] G. Schüürmann, R.-U. Ebert, R. Kühne, Chimia, 2006, 60, xxx.
[24] S. Canonica, J.B. Kramer, D. Reiss, H Gygax, Environ. Sci. Technol. 1997, 31, 1754.

[25] J.B. Kramer, S. Canonica, J. Hoigné, J. Kaschig, Environ. Sci. Technol. 1996, 30 , 2227.

[26] T. Poiger, F.G. Kari, W. Giger, Environ. Sci. Technol. 1999, 33, 533.

[27] J.M.A. Stoll, M.M. Ulrich, W. Giger, Environ. Sci. Technol. 1998, 32, 1875.

[28] J.W. Chen, W.J.G.M. Peijnenburg, X. Quan, Y.Z. Zhao, D.M. Xue, F.L. Yang, Chemosphere 1998, 37, 1169.

[29] C. Richard, S. Canonica, 'Aquatic phototransformation of organic contaminants induced by coloured dissolved natural organic matter', in 'The Handbook of Environmental Chemistry', Ed. O. Hutzinger, 2005, Springer: Berlin, Germany, p. 299.

[30] S. Canonica, P.G. Tratnyek, Environ. Toxicol. Chem. 2003, 22, 1743 .

[31] G.V. Buxton, C.L. Greenstock, W.P. Helman, A.B. Ross, J. Phys. Chem. Ref. Data 1988, 17,513

[32] M.M. Huber, S. Canonica, G.Y. Park, U. Von Gunten, Environ. Sci. Technol. 2003, 37, 1016.

[33] S. Canonica, U. Jans, K. Stemmler, J. Hoigné, Environ. Sci. Technol. 1995, 29, 1822.

[34] A.C. Gerecke, S. Canonica, S.R. Müller, M. Schärer, R.P. Schwarzenbach, Environ. Sci. Technol. 2001, 35, 3915.

[35] S. Canonica, B. Hellrung, J. Wirz, J. Phys. Chem. A 2000, 104, 1226.

[36] S. Canonica, B. Hellrung, P. Müller, J. Wirz, Environ. Sci. Technol. 2006, submitted.

[37] J. Hoigné, 'Formulation and calibration of environmental reaction kinetics: Oxidations by aqueous photooxidants as an example', in 'Aquatic Chemical Kinetics; Reaction Rates of Processes in Natural Waters', Ed. W. Stumm, 1990, Wiley-Interscience: New York. p. 43.

[38] R.S. Boethling, D.G. Lynch, J.S. Jaworska, J.L. Tunkel, G.C. Thom, S. Webb, Environ. Toxicol. Chem. 2004, 23, 911.

[39] P.H. Howard, Environ. Toxicol. Chem. 1985, 4, 129.

[40] P.H. Howard, Environ. Toxicol. Chem. 1993, 12, 1135 .

[41] J.S. Jaworska, R.S. Boethling, P.H. Howard, Environ. Toxicol. Chem. 2003, 22, 1710.

[42] J. Arnot, T. Gouin, D. Mackay, 'Practical Methods for Estimating Environmental Biodegradation Rates', 2005, Canadian Environmental Modelling Network: Peterborough.

[43] L. Gasser, K. Fenner, M. Scheringer, Environ. Sci. Technol. 2006, submitted.

[44] For this purpose, the difference in BIOWIN output between parent pesticide and transformation product was translated into a half-life difference using either the Arnot et al. [44] or our own regression. This halflife difference was then added directly to the experimental half-life of the parent compound to predict transformation product half-lives. The summary results of this approach are also given in Table 4.

[45] D. Aronson, R.S. Boethling, P.H. Howard, W. Stiteler, Chemosphere 2006, 63, 1953.

[46] K. Fenner, V. Lanz, M. Scheringer, M. Borsuk, Environ. Sci. Technol. 2006, submitted.

[47] G.D. Veith, SAR QSAR Environ. Res. 2004 $15,323$.

[48] A.P. van Wezel, A. Opperhuizen, Crit. Rev. Toxicol. 1995, 25, 255.

[49] C.L. Russom, S.P. Bradbury, S.J. Broderius, D.E. Hammermeister, R.A. Drummond, Environ. Toxicol. Chem. 1997, 16, 948.

[50] S.P. Bradbury, SAR QSAR Environ. Res. 1994, 2, 89.

[51] S. Basak, G. Grunwald, G. Host, G. Niemi, S. Bradbury, Environ. Toxicol. Chem. 1998, 17, 1056.

[52] G. Schüürmann, A.O. Aptula, R. Kühne, R.-U. Ebert, Chem. Res. Toxicol. 2003, 16, 974.

[53] S. Spycher, M. Nendza, J. Gasteiger, QSAR Comb. Sci. 2004, 23, 779.

[54] S. Spycher, 'QSARs for the Classification of Compounds into Modes of Toxic Action', 2005, PhD thesis, ComputerChemie-Centrum Erlangen, Universität Erlangen-Nürnberg, Erlangen. http:// www.opus.ub.uni-erlangen.de/opus/volltexte/2005/249/pdf/ SimonSypcherDissertation.pdf

[55] M.T.D. Cronin, T.W. Schultz, J. Mol. Struct. 2003, 622, 39 .

[56] A. Harder, B.I. Escher, P. Landini, N.B Tobler, R.P. Schwarzenbach, Environ. Sci. Technol. 2003, 37, 4962.

[57] A. Harder, B.I. Escher, R.P. Schwarzenbach, Environ. Sci. Technol. 2003, 37, 4955.

[58] P.C. von der Ohe, R. Kühne, R.-U. Ebert R. Altenburger, M. Liess, G. Schüürmann, Chem. Res. Toxicol. 2005, 18, 536.

[59] OECD, Guidance Document for Aquatic Effect Assessment, 1995, Organization for Economic Co-Ordination and Development, Paris.

[60] B. Escher, R.P. Schwarzenbach, Aquat. Sci. 2002, 64, 20.

[61] B.I. Escher, M. Snozzi, K. Häberli, R.P. Schwarzenbach, Environ. Toxicol. Chem. 1997, 16, 405

[62] R.W. Hunziker, B.I. Escher, R.P. Schwarzenbach, Environ. Toxicol. Chem. 2002 21,1191

[63] S. Spycher, B.I. Escher, J. Gasteiger, Chem. Res. Toxicol. 2005, 18, 1858.

[64] OECD, Report from the Expert Group on (Quantitative) Structure-Activity Relationships ((Q)SARs) on the Principles for the Validation of (Q)SARs, in OECD Environmental Health and Safety Publications, 2004, Organization for Economic Co-Ordination and Development: Paris. 